\title{
Order-Unity Correction to Hawking Radiation
}

\author{
Eanna E. Flanagan $\odot^{*}$ \\ Department of Physics, Cornell University, Ithaca, New York 14853, USA \\ and Cornell Laboratory for Accelerator-Based Sciences and Education (CLASSE), Cornell University, \\ Ithaca, New York 14853, USA
}

(Received 14 February 2021; revised 3 June 2021; accepted 22 June 2021; published 22 July 2021)

When a black hole first forms, the properties of the emitted radiation as measured by observers near future null infinity are very close to the 1974 prediction of Hawking. However, deviations grow with time and become of order unity after a time $t \sim M_{i}^{7 / 3}$, where $M_{i}$ is the initial mass in Planck units. After an evaporation time, the corrections are large: the angular distribution of the emitted radiation is no longer dominated by low multipoles, with an exponential falloff at high multipoles. Instead, the radiation is redistributed as a power-law spectrum over a broad range of angular scales, all the way down to the scale $\Delta \theta \sim 1 / M_{i}$, beyond which there is exponential falloff. This effect is a quantum gravitational effect, whose origin is the spreading of the wave function of the black hole's center-of-mass location caused by the kicks of the individual outgoing quanta, discovered by Page in 1980. The modified angular distribution of the Hawking radiation has an important consequence: the number of soft hair modes that can effectively interact with outgoing Hawking quanta increases from the handful of modes at low multipoles $l$ to a large number of modes, of order $\sim M_{i}^{2}$. We argue that this change unlocks the Hawking-Perry-Strominger mechanism for purifying the Hawking radiation.

DOI: 10.1103/PhysRevLett.127.041301

Introduction.-In the half century since its discovery, the Hawking evaporation of black holes and its associated conundrums have proved to be a fertile source of insights and progress in quantum gravity, from black hole thermodynamics to holography to links between quantum information and geometry [1-3]. At the same time, unresolved theoretical tensions have led to repeated scrutiny of the robustness of Hawking's predictions. An evaporating black hole is characterized by the small dimensionless parameter $1 / M$, where $M$ is the mass in Planck units, and there are small corrections that are perturbative in $1 / M$, as well as smaller corrections nonperturbative in $1 / M$. Large corrections, however, have been elusive.

There is a subtlety in classifying the size of corrections to Hawking radiation, related to the fact that the number of relevant field modes $N \sim M^{2}$ is large, and fractional corrections to expected values may be small for certain classes of operators but large for other operators. Suppose we decompose the Hilbert space $\mathcal{H}$ of radiation states at future null infinity $\mathscr{I}^{+}$as the product $\mathcal{H}=\mathcal{H}_{n} \otimes \mathcal{H}_{n}^{\prime}$, where $\mathcal{H}_{n}$ is the Hilbert space of a certain set of $n$ modes

Published by the American Physical Society under the terms of the Creative Commons Attribution 4.0 International license. Further distribution of this work must maintain attribution to the author(s) and the published article's title, journal citation, and DOI. Funded by SCOAP . with $n \leq N$. Given a correction $\Delta \rho$ to the density matrix $\rho$ on $\mathcal{H}$, we define

$$
\varepsilon_{\mathcal{H}_{n}}=\left\|\operatorname{tr}_{\mathcal{H}_{n}^{\prime}} \Delta \rho\right\|,
$$

where $\|A\|=\operatorname{tr} \sqrt{A^{\dagger} A}$, which gives a measure of the size of the correction to the state when restricted to $\mathcal{H}_{n}$. There exist perturbations $\Delta \rho$ for which $\varepsilon_{\mathcal{H}_{n}}$ is small whenever $n \ll N$, but for which $\varepsilon_{\mathcal{H}_{n}}$ is nevertheless of order unity when $n \sim N$. Such corrections have long been anticipated for Hawking radiation, since an order-unity correction to an entanglement entropy is required [4] for unitarity of the evaporation process $[2,3]$. Indeed, recent calculations using Euclidean path integrals have explicitly shown that the time evolution of the entanglement entropy of the Hawking radiation and the black hole is consistent with unitarity [611]. Hence there are corrections to Hawking radiation that are of order unity, for operators that involve $\sim N$ modes, although the new computational techniques do not yet allow computation of the corrected state.

In this Letter, we confine attention to operators that act on $n \ll N$ modes, for which the general expectation has been that corrections to Hawking radiation are small. We show that there are corrections at the level of individual modes that are of order unity, arising from quantum gravitational effects in the deep infrared. The mechanism is straightforward: secularly increasing fluctuations in the center-of-mass location of the black hole cause a change in 
the angular distribution of the radiation, with most of the power being redistributed to small angular scales. Although the modifications to the radiation do not directly impact the issue of how unitarity of the evaporation is achieved, we will argue that there is an important indirect effect.

In the remainder of the Letter, we first give a heuristic argument for the effect, then give a detailed derivation, and conclude with a discussion of some implications. Throughout we use Planck units with $G=\hbar=c=1$.

Redistribution of power to small angular scales: Brief heuristic argument.-As described by Page [12], the emission of Hawking radiation causes the uncertainty in the black hole's center of mass to grow with time. This growth is easy to understand: each outgoing quantum carries off a momentum $\sim M^{-1}$ in a random direction, and the resulting perturbation to the velocity of the black hole is of order $\sim M^{-2}$. Over an evaporation time $\sim M^{3}$, this single kick yields a displacement of the center-of-mass position of the black hole of order $\sim M$. Over the course of the evaporation process, we have $N \sim M^{2}$ such kicks that accumulate as a random walk, giving a total net uncertainty in the position of the black hole of order $\sim \sqrt{N} M \sim M^{2}$.

Now if a black hole displaced by $\sim M^{2}$ emits a single quantum in a wave packet mode of duration $\sim M$, the energy flux at future null infinity $\mathscr{I}^{+}$is delayed by $\sim M^{2}$ on

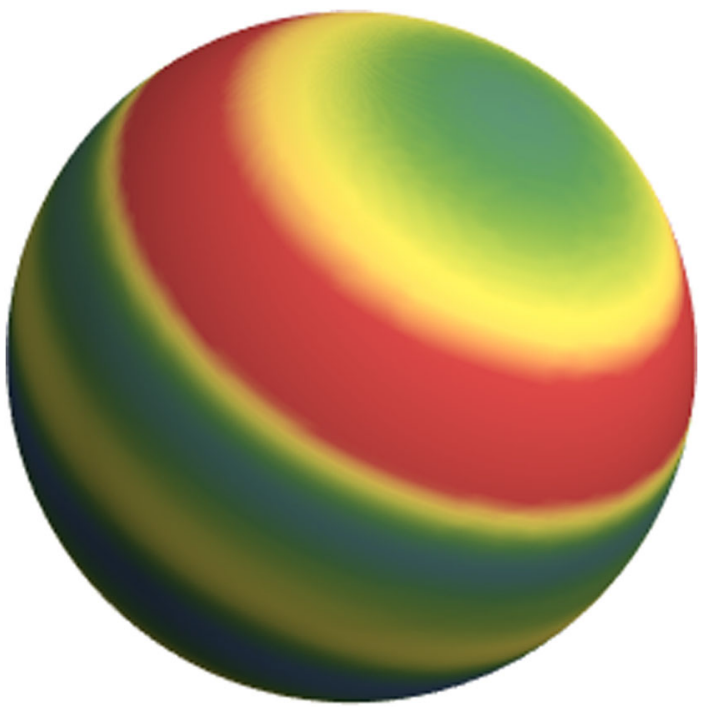

FIG. 1. An illustration of the standard Unruh state of an evaporating nonspinning black hole, at a particular instant of retarded time at future null infinity, in a reference frame that is displaced from the black hole center of mass by several Schwarzschild radii. The quantity plotted is a typical realization of the Gaussian random process on the sphere whose two-point function is given by taking the two-point function of a scalar field in the Unruh state at future null infinity and subtracting the twopoint function of the out vacuum. Fluctuations in individual wave packet modes give rise to fluctuations on the sphere that are confined to concentric thin strips, giving rise to a characteristic angular scale that is small compared to unity. one side of $\mathscr{I}^{+}$and advanced on the other. On a cut of fixed retarded time, the energy flux due to this quantum will be localized to a thin strip on the sphere of width $\Delta \theta \sim$ $M / M^{2} \sim 1 / M$ (see Fig. 1), and so the power spectrum of the radiation as a function of angular scale will be peaked at angular scales $\sim 1 / M$.

Redistribution of power to small angular scales: Derivation.-Although the mechanism that modifies the Hawking radiation is universal, for simplicity, we will specialize here to a four-dimensional Schwarzschild black hole coupled to a massless free scalar field $\Phi$. Near $\mathscr{I}^{+}$we use retarded Bondi coordinates $(u, r, \theta, \phi)$. We resolve the Bondi-Metzner-Sachs (BMS) transformation freedom in these coordinates by choosing the canonical coordinates [13] associated with the approximate stationary state of the black hole shortly after it is formed at $u=0$, say, before it has time to emit appreciable amounts of Hawking radiation [15]. This choice also determines a particular Poincaré subgroup of the BMS group.

We define the field $\varphi$ on $\mathscr{I}^{+}$by

$$
\Phi(u, r, \theta, \phi)=\frac{\varphi(u, \theta, \phi)}{r}+O\left(\frac{1}{r^{2}}\right)
$$

and we denote by $\mathcal{H}$ the Hilbert space of out-states on $\mathscr{I}^{+}$ parametrized by $\varphi$. We denote by $M_{i}$ the initial mass of the black hole at $u=0$, and by $M=M\left(u_{1}\right)<M_{i}$ the Bondi mass at some later retarded time $u_{1}$ with $u_{1} \gg M_{i}$. We denote by $\rho_{U}$ the Hawking radiation state on $\mathcal{H}$ for an eternal black hole of mass $M$, i.e., the Unruh vacuum. In the standard calculation, it is argued that this state provides a good approximation to the $n$-point functions on $\mathscr{I}^{+}$of the state for the gravitational collapse spacetime, for retarded times $u$ with $\left|u-u_{1}\right|$ small compared to the evaporation time $M^{3}$.

For any density matrix $\rho$ on $\mathcal{H}$, we define the regularized two-point function

$$
\begin{aligned}
G\left(u, \boldsymbol{\theta} ; u^{\prime}, \boldsymbol{\theta}^{\prime}\right)= & \operatorname{tr}\left[\rho \varphi(u, \boldsymbol{\theta}) \varphi\left(u^{\prime}, \boldsymbol{\theta}^{\prime}\right)\right] \\
& -{ }_{\text {out }}\left\langle 0\left|\varphi(u, \boldsymbol{\theta}) \varphi\left(u^{\prime}, \boldsymbol{\theta}^{\prime}\right)\right| 0\right\rangle_{\text {out }},
\end{aligned}
$$

where $|0\rangle_{\text {out }}$ is the out vacuum and $\boldsymbol{\theta}=(\theta, \phi)$. For stationary, spherically symmetric states, we have $G=G(\Delta u, \gamma)$, where $\Delta u=u-u^{\prime}$ and $\gamma$ is the angle between $\boldsymbol{\theta}$ and $\boldsymbol{\theta}^{\prime}$. We define the Fourier transform $\tilde{G}(\omega, \gamma)=\int d \Delta u e^{i \omega \Delta u} G(\Delta u, \gamma)$, and decompose this in angular harmonics as

$$
\tilde{G}(\omega, \gamma)=\sum_{l=0}^{\infty} \frac{2 l+1}{4 \pi} P_{l}(\cos \gamma) S(\omega, l) .
$$

The quantity $S(\omega, l)$ is related to the energy flux $\dot{E}$ to infinity per unit frequency $\omega$ in field multipoles [16] of order $l$ by 


$$
\left(\frac{d \dot{E}}{d \omega}\right)_{l}=\frac{2 l+1}{2 \pi} \omega^{2} S(\omega, l) .
$$

We denote by $G_{U}$ the regularized two-point function of the Unruh vacuum, for which the corresponding energy flux is

$$
\left(\frac{d \dot{E}}{d \omega}\right)_{U, l}=\frac{2 l+1}{2 \pi} \frac{\omega\left|t_{l \omega}\right|^{2}}{e^{\beta \omega}-1} .
$$

Here $t_{l \omega}$ is the transmission coefficient through the effective potential and $\beta=8 \pi M$ is the inverse temperature of the radiation. As is well known, most of the power in the spectrum (6) is concentrated at $l \sim O(1)$, with an exponential falloff at large $l$.

We now want to derive how the energy spectrum (6) as a function of frequency and angular scale is modified. The key idea is to supplement the standard computation by including the evolution of a small number of relevant infrared gravitational degrees of freedom, specifically the BMS charges as computed on cuts $u=$ const of $\mathscr{I}^{+}$. In the classical theory, the values of these charges determine the spacetime geometry when the black hole is stationary, and we assume that this is still true in the quantum theory when both the charges and geometry have quantum fluctuations. We focus, in particular, on the black hole's center of mass $\Delta$, encoded in the orbital angular momentum associated with the Poincare subgroup of the BMS group discussed above.

The framework we use is anchored at $\mathscr{I}^{+}$, where the coordinate system $(u, \theta, \varphi)$ and out Hilbert space $\mathcal{H}$ for the scalar field are unaffected by the large quantum fluctuations of the gravitational charges and of the geometry in the interior. As described above, the fluctuations in $\Delta$ grow with time due to repeated kicks from outgoing Hawking quanta. We divide $\mathscr{I}^{+}$into an early portion $\mathscr{I}_{\text {early }}^{+}$with $u<u_{1}$ and a late portion $\mathscr{I}_{\text {late }}^{+}$with $u>u_{1}$. The Hilbert space $\mathcal{H}$ can be correspondingly factored [17] into the tensor product $\mathcal{H}_{\text {early }} \otimes \mathcal{H}_{\text {late }}$. The state of the center of mass at time $u_{1}$ is strongly correlated with the Hawking radiation on $\mathscr{I}_{\text {early }}^{+}$, by momentum conservation for each emission event, and if we trace over $\mathcal{H}_{\text {early }}$ we obtain a mixed state for the center of mass [23]. This state can be described in terms of its Wigner function $\mathcal{W}(\boldsymbol{\Delta}, \mathbf{p})$, a function of the three-dimensional position $\Delta$ and momentum $\mathbf{p}$ of the black hole. Denoting a position eigenstate by $|\boldsymbol{\Delta}\rangle$, the corresponding state is

$$
\int d^{3} \Delta \int d^{3} \xi \tilde{\mathcal{W}}(\boldsymbol{\Delta}, \xi)|\boldsymbol{\Delta}-\xi / 2\rangle\langle\boldsymbol{\Delta}+\xi / 2|,
$$

where $\tilde{\mathcal{W}}(\boldsymbol{\Delta}, \xi)=\int d^{3} p \exp [-i \mathbf{p} \cdot \xi] \mathcal{W}(\boldsymbol{\Delta}, \mathbf{p})$. Since the kicks from the individual outgoing quanta are uncorrelated, the Wigner function $\mathcal{W}$ is very nearly Gaussian by the multivariate central limit theorem. Hence $\mathcal{W}$ has the form
$\tilde{\mathcal{W}}(\boldsymbol{\Delta}, \xi)=\mathcal{N} \exp \left[-\frac{1}{2} \frac{\Delta^{2}}{\sigma_{\Delta}^{2}}-\frac{1}{2}\left(1-\varepsilon^{2}\right) \xi^{2} \sigma_{p}^{2}-i \varepsilon \frac{\sigma_{p}}{\sigma_{\Delta}} \Delta \cdot \xi\right]$

where $\mathcal{N}=(2 \pi)^{-3 / 2} \sigma_{\Delta}^{-3}$, the quantities $\sigma_{\Delta}^{2}$ and $\sigma_{p}^{2}$ are the variances in position and momentum, and $\varepsilon$ with $|\varepsilon|<1$ is a correlation coefficient. The evolution of these parameters is studied in Ref. [25], which shows that $\varepsilon$ is of order unity and

$\begin{aligned} \sigma_{\Delta}^{2} & = \begin{cases}c_{0} M_{i}^{4}\left(1-M^{3} / M_{i}^{3}\right)^{3} & M_{i}-M \ll M_{i}, \\ c_{1} M_{i}^{4} & \sqrt{M_{i}} \ll M \ll M_{i},\end{cases} \\ \sigma_{p}^{2} & =c_{2} \ln \left(M_{i} / M\right) \quad \sqrt{M_{i}} \ll M,\end{aligned}$

where $c_{0}, c_{1}$, and $c_{2}$ are dimensionless constants of order unity.

We now turn to describing how the fluctuations in the center of mass of the black hole affect the Hawking radiation. In Minkowski spacetime we can define a displacement operator $U_{\Delta}$, which displaces any state by an amount $\Delta$, which acts on the field operator according to $U_{\Delta}^{\dagger} \Phi(t, \mathbf{r}) U_{\Delta}=\Phi(t, \mathbf{r}-\boldsymbol{\Delta})$. This operator extends naturally to the Hilbert space $\mathcal{H}$ of out-states on the black hole spacetime, where its action is defined by

$$
U_{\Delta}^{\dagger} \varphi(u, \boldsymbol{\theta}) U_{\Delta}=\varphi(u+\mathbf{n} \cdot \boldsymbol{\Delta}, \boldsymbol{\theta}),
$$

with $\mathbf{n}$ the unit vector in the direction specified by $\boldsymbol{\theta}$. The Unruh state for a black hole displaced from the origin by an amount $\Delta$ can be written as

$$
|\mathbf{\Delta}\rangle \otimes \sum_{j}\left|\chi_{j}\right\rangle U_{\Delta}\left|\psi_{j}\right\rangle
$$

where $\left|\chi_{j}\right\rangle$ is a set of states on the future horizon and $\left|\psi_{j}\right\rangle$ is a set of states in $\mathcal{H}$. Taking the trace over the horizon states gives for the corresponding Unruh state at $\mathscr{I}^{+}$

$$
|\boldsymbol{\Delta}\rangle\langle\boldsymbol{\Delta}| \otimes U_{\Delta} \rho_{U} U_{\Delta}^{\dagger},
$$

where $\rho_{U}=\sum_{j} c_{j}^{2}\left|\psi_{j}\right\rangle\left\langle\psi_{j}\right|$ with $c_{j}^{2}=\left\langle\chi_{j} \mid \chi_{j}\right\rangle$.

Suppose now that the state of the black hole's center of mass were fixed and not evolving with time, given by Eq. (7) for the fixed values of the parameters $\sigma_{\Delta}, \sigma_{p}$, and $\varepsilon$ evaluated at $u=u_{1}$. Then by linearity from Eqs. (7), (11), and (12), the corresponding out-state would be

$$
\int d^{3} \Delta \int d^{3} \xi \tilde{\mathcal{W}}(\boldsymbol{\Delta}, \xi)|\boldsymbol{\Delta}-\xi / 2\rangle\langle\boldsymbol{\Delta}+\xi / 2| \otimes U_{\Delta-\xi / 2} \rho_{U} U_{\Delta+\xi / 2}^{\dagger} .
$$

Tracing over the center-of-mass Hilbert space gives the corrected version of the Unruh state 


$$
\rho_{U, \mathrm{corr}}=\int d^{3} \Delta \tilde{\mathcal{W}}(\boldsymbol{\Delta}, \mathbf{0}) U_{\Delta} \rho_{U} U_{\Delta}^{\dagger} .
$$

Of course the state of the center of mass is evolving with time and not fixed. Nevertheless, the corrected Unruh state (14) should give a good approximation to the $n$-point functions on $\mathscr{I}^{+}$of the field at retarded times $u$ that satisfy two conditions: (i) We have $\left|u-u_{1}\right| \ll M^{3}$, so the mass of the black hole as well as the state of the center-of-mass have not evolved significantly from their values at $u=u_{1}$. (ii) We have $u-u_{1} \gg \sigma_{\Delta} \sim M_{i}^{2}$. This ensures that the displacements (10) in retarded time caused by the operators $U_{\Delta}$ in Eq. (14) do not generate a dependence on degrees of freedom on $\mathscr{I}_{\text {early }}^{+}$, which we have already traced over to compute the state (7).

We now turn to showing that the modifications inherent in the corrected Unruh state (14) are of order unity, for individual outgoing wave packet modes at sufficiently late times. Combining Eqs. (3), (10), and (14) gives for the regularized two-point function of the corrected Unruh state

$$
\begin{aligned}
G_{U, \text { corr }}\left(u, \boldsymbol{\theta} ; u^{\prime}, \boldsymbol{\theta}^{\prime}\right)= & \int d^{3} \Delta \tilde{\mathcal{W}}(\boldsymbol{\Delta}, \mathbf{0}) \\
& \times G_{U}\left(u+\mathbf{n} \cdot \boldsymbol{\Delta}, \boldsymbol{\theta} ; u^{\prime}+\mathbf{n}^{\prime} \cdot \boldsymbol{\Delta}, \boldsymbol{\theta}^{\prime}\right),
\end{aligned}
$$

using that the Wightman function in the second term in Eq. (3) is invariant under translations. The corresponding functions of frequency $\omega$ and angle $\gamma$ are related by

$$
\begin{aligned}
\tilde{G}_{U, \text { corr }}(\omega, \gamma) & =\int d^{3} \Delta \tilde{\mathcal{W}}(\boldsymbol{\Delta}, \mathbf{0}) e^{-i \omega\left(\mathbf{n}-\mathbf{n}^{\prime}\right) \cdot \Delta} \tilde{G}_{U}(\omega, \gamma) \\
& =\exp \left[-2 \omega^{2} \sigma_{\Delta}^{2} \sin ^{2}(\gamma / 2)\right] \tilde{G}_{U}(\omega, \gamma),
\end{aligned}
$$

where we have used Eq. (8). Note that the transformation (16) preserves $\tilde{G}(\omega, 0)$, which is proportional to the total energy flux per unit frequency, summed over all multipoles. Hence the transformation redistributes power over angular scales, but not from one frequency to another.

We next combine Eqs. (4), (5), and (16) to obtain for the spectrum of outgoing radiation

$$
\left(\frac{d \dot{E}}{d \omega}\right)_{U, \text { corr }, l}=(2 l+1) \omega^{2} \int_{-1}^{1} d \mu P_{l}(\mu) e^{-\omega^{2} \sigma_{\Delta}^{2}(1-\mu)} \tilde{G}_{U}(\omega, \gamma),
$$

where $\mu=\cos \gamma$. We now specialize to frequencies of the order $\omega \sim M^{-1}$, where most of the outgoing power is located. We thus exclude high frequencies $\omega \gg M^{-1}$, where the power is exponentially suppressed, and low frequencies $\omega \ll M^{-1}$, where it is power-law suppressed, from the spectrum (6). Since the function $\omega \tilde{G}_{U}(\omega, \gamma)$ depends on $\omega$ and $M$ only through the combination $\omega M$
[26], which is of order unity, for such frequencies $\tilde{G}_{U}$ varies with $\gamma$ only on angular scales of order unity; there are no other dimensionless parameters on which the function depends. It follows that $\tilde{G}_{U}$ has negligible variation over the range $0 \leq \gamma \lesssim 1 /\left(\omega \sigma_{\Delta}\right) \sim M / M_{i}^{2} \ll 1$ that is not exponentially suppressed by the exponential factor in Eq. (17). Hence we can evaluate this function at $\gamma=0$ and pull it outside the integral, and using Eqs. (4) and (5) we reexpress it in terms of the total power per unit frequency $d \dot{E} / d \omega=\sum_{l}(d \dot{E} / d \omega)_{l}$ in the Unruh state. We evaluate the remaining integral using the identity $\int d \mu P_{l}(\mu) e^{a \mu}=$ $\sqrt{2 \pi / a} I_{l+1 / 2}(a)$, which expresses it terms of a modified Bessel function of the first kind [27]. The final result is

$$
\left(\frac{d \dot{E}}{d \omega}\right)_{U, \text { corr }, l}=\sqrt{\frac{\pi}{2}}\left(\frac{d \dot{E}}{d \omega}\right)_{U} \frac{(2 l+1) e^{-\omega^{2} \sigma_{\Delta}^{2}}}{\omega \sigma_{\Delta}} I_{l+1 / 2}\left(\omega^{2} \sigma_{\Delta}^{2}\right) .
$$

Using the approximate formula $I_{l+1 / 2}(a)=(2 \pi a)^{-1 / 2} e^{a}[1+$ $\left.O\left(l^{2} / a\right)\right]$, this simplifies to [28]

$$
\left(\frac{d \dot{E}}{d \omega}\right)_{U, \mathrm{corr}, l}=\left(\frac{d \dot{E}}{d \omega}\right)_{U} \frac{(2 l+1)}{2 \omega^{2} \sigma_{\Delta}^{2}}\left[1+O\left(\frac{l^{2}}{\omega^{2} \sigma_{\Delta}^{2}}\right)\right] .
$$

This corresponds to a power-law spectrum for angular scales in the range $0 \leq l \ll l_{\text {crit }}$ with $l_{\text {crit }}=\omega \sigma_{\Delta}$, with most of the power in the vicinity of $l \sim l_{\text {crit }}$. At scales $l \geq l_{\text {crit }}$ the spectrum falls off exponentially, from the upper bound $I_{l+1 / 2}(a) \leq(2 \pi a)^{-1 / 2} e^{a} \exp [-l /(4 \sqrt{a})]$ for $l \geq \sqrt{a} \gg 1$.

We now consider the critical angular scale $l_{\text {crit }}=\omega \sigma_{\Delta}$. At sufficiently late times $u \gtrsim M_{i}^{3}$, we have $\sigma_{\Delta} \sim M_{i}^{2}$ from Eq. (9), and so the critical angular scale is $l_{\text {crit }} \sim M_{i}^{2} / M \gg$ 1 using $\omega \sim M^{-1}$, which reduces to $\sim M_{i}$ if $M \sim M_{i}$. At early times, we have from Eq. (9) and using $u / M_{i}^{3} \sim 1-$ $M^{3} / M_{i}^{3}$ that $l_{\text {crit }} \sim u^{3 / 2} M_{i}^{-7 / 2}$, so the modification effect first becomes of order unity after an interval of retarded time $u \sim M_{i}^{7 / 3}$.

Discussion and conclusions.-We close with a number of comments. First, the modification to the Hawking radiation does not alter the amount of entanglement between modes inside the horizon and those outside, and so does not directly impact the unitarity of the evaporation process. The exterior modes that are relevant at late times depend, through the position of the black hole, on which early time exterior modes are occupied (the total number of relevant exterior modes has increased from $\sim M_{i}^{2}$ to $\sim M_{i}^{4}$ ). This effect generates nontrivial mutual information [2] between early Hawking radiation and late Hawking radiation, but does not alter the total entanglement between interior and exterior modes.

Second, the corrected Unruh state (14) is not a Gaussian state, unlike the original Unruh state, although it is 
stationary and spherically symmetric. Thus it is not determined by the two-point function (18), although it is completely determined by the formulas (8) and (14).

Third, the general mechanism discussed here involving spatial translations clearly also applies to other generators of the BMS group. The black hole at late times determines a BMS frame that is related to the initial BMS frame by a transformation which includes a rotation, boost, and supertranslation, and secularly growing quantum fluctuations in those transformations modify the outgoing Hawking radiation. However, in Ref. [25] we estimate that the typical boost [29] velocity scale is $\sim 1 / M$, and that the length scale involved in the supertranslation fluctuations is $\sim 1$, so the corresponding modifications to the Hawking radiation are small.

Fourth, consider the result of interpreting the corrected Unruh state (14) on $\mathscr{I}^{+}$in terms of a single semiclassical spacetime with the black hole at the origin. An outgoing mode with $l \sim M_{i}$ near $\mathscr{I}^{+}$corresponds near the black hole to a large amplitude standing wave in a thin shell of width $\sim 1$ in the nonevanescent region between the horizon and potential barrier, which varies over transverse length scales along the horizon of order $\sim 1$. This Planckian behavior of the extrapolated corrected Unruh state illustrates the potential pitfalls of thinking in terms of a single semiclassical spacetime and focusing on near-horizon physics.

Fifth, we argue that the modification to the Hawking process removes one of the primary objections to the proposal that soft hair on black holes plays a key role in resolving the information loss paradox [21,22,30-32]. Soft hair consists of charges measurable at future null infinity associated with an extension of the BMS group [33-36], higher- $l$ analogs of the center of mass that are encoded in the asymptotic metric. Just as for the center of mass, the expected value of soft hair charges can be set to zero by a gauge transformation, locally in time, but their variances cannot and can contain nontrivial information. Outgoing Hawking quanta excite soft hair via the gravitational wave memory effect. It has been suggested that the Hawking radiation is purified at late times by its entanglement with soft hair degrees of freedom [30].

A difficulty with this proposal has been that only low $l$ modes of the soft hair can be excited by the outgoing quanta, because of the exponential falloff of the spectrum (6) at high $l$. The soft hair field $\Phi(\boldsymbol{\theta})$ is given in terms of the scalar field $\varphi$ on $\mathscr{I}^{+}$by [see, e.g., Eqs. (2.19) and (4.4) of Ref. [14] ]

$$
D^{2}\left(D^{2}+2\right) \Phi(\boldsymbol{\theta})=32 \pi \mathcal{P} \int d u \varphi_{, u}(u, \boldsymbol{\theta})^{2},
$$

where $D^{2}$ is the Laplacian on the two-sphere and $\mathcal{P}$ is a projection operator that sets to zero $l=0,1$ modes. Thus only a handful of soft hair modes can be excited, too few to play a relevant role for purifying the $\sim M_{i}^{2}$ outgoing Hawking quanta.
The modified angular distribution of the Hawking radiation completely changes this picture, since the source term in Eq. (20) now extends effectively up to multipoles of order $l \sim M_{i}$. This makes $\sim M_{i}^{2}$ soft hair modes potentially accessible, enough for each outgoing quantum to interact with its own soft hair mode. Note, however, that this scenario cannot be analyzed within a single semiclassical spacetime. The details of the interaction of the Hawking radiation with the soft hair is an intriguing subject for further study.

I thank Abhay Ashtekar, Venkatesa Chandrasekaran, and Kartik Prabhu for helpful discussions and an anonymous referee for useful comments. This research was supported in part by NSF Grants No. PHY-1404105 and No. PHY1707800 .

*eef3@cornell.edu

[1] S. W. Hawking, Breakdown of predictability in gravitational collapse, Phys. Rev. D 14, 2460 (1976).

[2] D. Harlow, Jerusalem lectures on black holes and quantum information, Rev. Mod. Phys. 88, 015002 (2016).

[3] D. Marolf, The black hole information problem: Past, present, and future, Rep. Prog. Phys. 80, 092001 (2017).

[4] The fact that $\|\Delta \rho\|=O(1)$ is required follows from the identity $|S(\rho+\Delta \rho)-S(\rho)| \leq\|\Delta \rho\| \log d+1 / e$, where $S$ is von Neumann entropy and $d$ is the dimension of the Hilbert space [5], together with $S(\rho+\Delta \rho)=0$ and $S(\rho) \sim \log d \sim M^{2}$.

[5] M. A. Nielsen, Continuity bounds for entanglement, Phys. Rev. A 61, 064301 (2000).

[6] A. Almheiri, N. Engelhardt, D. Marolf, and H. Maxfield, The entropy of bulk quantum fields and the entanglement wedge of an evaporating black hole, J. High Energy Phys. 12 (2019) 063.

[7] G. Penington, Entanglement wedge reconstruction and the information paradox, J. High Energy Phys. 09 (2020) 002.

[8] A. Almheiri, R. Mahajan, J. Maldacena, and Y. Zhao, The Page curve of Hawking radiation from semiclassical geometry, J. High Energy Phys. 03 (2020) 149.

[9] A. Almheiri, T. Hartman, J. Maldacena, E. Shaghoulian, and A. Tajdini, Replica wormholes and the entropy of Hawking radiation, J. High Energy Phys. 05 (2020) 013.

[10] G. Penington, S. H. Shenker, D. Stanford, and Z. Yang, Replica wormholes and the black hole interior, arXiv: 1911.11977.

[11] A. Almheiri, T. Hartman, J. Maldacena, E. Shaghoulian, and A. Tajdini, The entropy of Hawking radiation, arXiv: 2006.06872.

[12] D. N. Page, Is Black-Hole Evaporation Predictable?, Phys. Rev. Lett. 44, 301 (1980).

[13] See, for example, Sec. II. C of Ref. [14].

[14] E. E. Flanagan and D. A. Nichols, Conserved charges of the extended Bondi-Metzner-Sachs algebra, Phys. Rev. D 95, 044002 (2017).

[15] Note that imposing this requirement in the region of $\mathscr{I}^{+}$ near $u=0$ determines the coordinates everywhere on $\mathscr{I}^{+}$because of the properties of the BMS group. 
[16] Note that there are two different methods of defining the angular spectrum of Hawking radiation. One can decompose the field into spherical harmonic modes or decompose the stress energy tensor. We use the former definition. The two definitions are not equivalent since the stress energy tensor depends nonlinearly on the field. Nevertheless, the qualitative result that power is redistributed from large angular scales to small angular scales will clearly be be valid for both definitions.

[17] This is not quite true, as there are also "edge modes" associated with the boundary $u=u_{1}$ [18-20], equivalent to soft hair [21,22]. We neglect these modes here as they are not relevant to the present discussion, but will return to them later in the Letter.

[18] W. Donnelly and L. Freidel, Local subsystems in gauge theory and gravity, J. High Energy Phys. 09 (2016) 102.

[19] A. J. Speranza, Local phase space and edge modes for diffeomorphism-invariant theories, J. High Energy Phys. 02 (2018) 021.

[20] M. Geiller, Edge modes and corner ambiguities in 3d ChernSimons theory and gravity, Nucl. Phys. B924, 312 (2017).

[21] S. W. Hawking, M. J. Perry, and A. Strominger, Soft Hair on Black Holes, Phys. Rev. Lett. 116, 231301 (2016).

[22] S. W. Hawking, M. J. Perry, and A. Strominger, Superrotation charge and supertranslation hair on black holes, J. High Energy Phys. 05 (2017) 161.

[23] One can think of this as the early Hawking radiation decohering the black hole location [24].

[24] A. Arrasmith, A. Albrecht, and W. H. Zurek, Decoherence of black hole superpositions by Hawking radiation, Nat. Commun. 10, 1024 (2019).

[25] E. E. Flanagan, Infrared effects in the late stages of black hole evaporation, arXiv:2102.13629.
[26] F. Gray and M. Visser, Greybody factors for Schwarzschild black holes: Path-ordered exponentials and product integrals, Universe 4, 93 (2018).

[27] F. W. Olver, D. W. Lozier, R. F. Boisvert, and C. W. Clark, NIST Handbook of Mathematical Functions, 1st ed. (Cambridge University Press, Cambridge, 2010), formula 18.17.19.

[28] For $l \gg 1$ Eq. (19) can be more simply derived by approximating the sphere as a plane and replacing the transform (17) with a two-dimensional Fourier transform.

[29] The effect of boosts is also suppressed by the fact that the operator describing the evolution of the quantum field is approximately diagonal on a position basis for the black hole center of mass, but not on a momentum basis. This is why boost fluctuations are not present in Eq. (14), although they are present in the Wigner function $\mathcal{W}$.

[30] A. Strominger, Black hole information revisited, arXiv: 1706.07143.

[31] S. Pasterski and H. Verlinde, HPS meets AMPS: How soft hair dissolves the firewall, arXiv:2012.03850.

[32] P. Cheng and Y. An, Soft black hole information paradox: Page curve from Maxwell soft hair of black hole, Phys. Rev. D 103, 126020 (2021).

[33] A. Strominger, Lectures on the Infrared Structure of Gravity and Gauge Theory (Princeton University Press, Princeton, NJ, 2018).

[34] M. Campiglia and A. Laddha, Asymptotic symmetries and subleading soft graviton theorem, Phys. Rev. D 90, 124028 (2014).

[35] M. Campiglia and A. Laddha, New symmetries for the gravitational S-matrix, J. High Energy Phys. 04 (2015) 076.

[36] G. Compère, A. Fiorucci, and R. Ruzziconi, Superboost transitions, refraction memory and super-Lorentz charge algebra, J. High Energy Phys. 11 (2018) 200. 